\title{
A Histogram Curve-matching Approach for Object-based Image Change Analysis of Urban Land Use
}

Gl_Forum 2020, Issue 2

Page: 160 - 174

Full Paper

Corresponding Author:

stow@sdsu.edu

DOI: 10.1553/giscience2020_02_s160

\author{
Chi-Feng Yen', Douglas Alan Stow' and Sory Toure ${ }^{2}$ \\ ISan Diego State University, USA \\ ${ }^{2}$ Consolidated Safety Services, Inc., USA
}

\begin{abstract}
Geographic object-based image analysis (GEOBIA) is commonly applied for land-cover and land-use mapping, updating and change-identification analyses. Following image segmentation, conventional GEOBIA routines classify image objects based on parametric statistical measures, assuming that within-object pixels have normally distributed image brightness signatures. The context for this study is updating extant land-use GIS layers that are out of date as a result of urban expansion. The objective is to develop, test and compare GEOBIA techniques based on a histogram classifier and on a nearest-neighbour classifier, for updating land-use layers. Frequency distribution signatures of land-use change and nochange objects are evaluated for different feature inputs and classifiers within an urbanizing area in San Diego County, California, USA. The results demonstrate that a histogram classifier consistently outperforms a conventional nearest-neighbour classifier. A Histogram Matching Root Sum Squared Differential Area classifier combined with temporal-spectral difference inputs and arithmetic mean for combining multi-feature classifier metrics yielded the greatest accuracy: $79.82 \%$ overall accuracy, with $78.72 \%$ and $81.07 \%$ for change and nochange objects respectively.
\end{abstract}

\section{Keywords:}

land-use change identification, urban growth, GEOBIA, histogram matching

\section{Introduction}

With the greater availability of high spatial resolution (H-res) remote sensing imagery, geographic object-based image analysis (GEOBIA) has been widely adopted for land-cover and land-use mapping and change analyses (Blaschke et al. 2014). GEOBIA is commonly more accurate than traditional per-pixel-based classification and change-identification approaches because it combines individual pixels into image objects (aka segments), which are subsequently classified based on spectral or contextual criteria with statistical (e.g. mean and standard deviation) or rule-based measurements (Cleve et al. 2008; Gao and Mas 2008; Whiteside, Boggs and Maier 2011). Conventional parametric statistical measurements assume 
that the within-object pixels are normally distributed and may ignore the richness of frequency distribution signatures of within-object pixels, particularly for objects derived from H-res imagery of urban scenes having spatially heterogeneous material compositions. A few studies have taken advantage of the shape characteristics of within-object histograms for landcover/land-use (LCLU) classification based on a single-date H-res image (Stow et al. 2012; Toure et al. 2013). However, the curve-matching approach for classification of temporalspectral objects has not been tested prior to this study.

The growing development of GEOBIA techniques facilitates research on delineating and identifying land-change objects using geographic object-based image change analysis (GEOBICA) (Stow 2010). Many studies demonstrate that GEOBICA outperforms traditional per-pixel-based change identification when applied to multi-temporal H-res imagery for landuse/land-cover change analysis (Chen et al. 2012; Hussain et al. 2013; Johansen et al. 2010). Desclée, Bogaert and Defourny (2006) segmented and classified a multi-temporal SPOT-HRV image layerstack consisting of three layers of reflectance difference bands. The resultant forest land-cover change map yielded high detection accuracy $(>90 \%)$ and overall kappa $(>0.80)$.

GEOBIA approaches to land-cover and land-use change (LCLUC) analysis can be categorized as (1) post-classification comparison or (2) multi-temporal image layerstack (Stow 2010). For post-classification comparison, individual GEOBIA classifications are performed, and then 'from-to' LCLUC (e.g. bare soil to residential built) are mapped by map overlay. Zhou et al. (2008) and King (2011) applied post-classification comparison to GEOBICA for urban landuse/land-cover changes with H-res aerial imagery and achieved substantially greater changeidentification accuracy in the resultant LCLUC maps compared to those created by a pixelbased approach. Multi-temporal image layerstack classification takes advantage of multitemporal transitions to identify LCLUC from co-registered image objects in single segmentation and classification processes. Im et al. (2008) utilized object/neighbourhood correlation images and segmentations of the bi-temporal correlation image layerstack to delineate and identify land-use/land-cover changes.

The objectives of this study are to test and compare land-use change (LUC) identification methods based on a histogram curve-matching classification. The histogram curve-matching classification results are compared to products generated by the standard nearest-neighbour classifier. This change-identification procedure is tested for a study area in southern San Diego County, California, based on multispectral aerial orthoimages having $1 \mathrm{~m}$ spatial resolution. The application context for this research is the semi-automatic updating of existing land-use GIS layers that become out of date following urban growth.

The following research questions are examined as part of the research:

1. Do LUC and no-change objects have non-normally distributed histogram curves when extracted from a multi-temporal H-res image layerstack captured for an urbanizing area?

2. Does the classification of temporal-spectral LUC objects based on histogram curvematching demonstrate potential to improve classification accuracy relative to traditional nearest-neighbour classifiers?

3. Which feature inputs to a histogram-matching classifier yield the highest overall agreement when identifying LUC / no-change objects? 


\section{Study Area and Data}

Figure 1 shows a bi-temporal aerial orthoimage layerstack covering a $266 \mathrm{~km} 2$ study area that encompasses portions of the cities of National City, Chula Vista and San Diego, in San Diego County, California, USA. The geographic coordinates of the study area are $32^{\circ} 41^{\prime} 07^{\prime \prime}$ to $32^{\circ} 33^{\prime} 20^{\prime \prime} \mathrm{N}$ and $117^{\circ} 05^{\prime} 19^{\prime \prime}$ to $116^{\circ} 53^{\prime} 41^{\prime \prime} \mathrm{W}$. In the last two decades, this area has experienced substantial LU change, especially from vacant and undeveloped land to new single-family and multi-family residential land use.

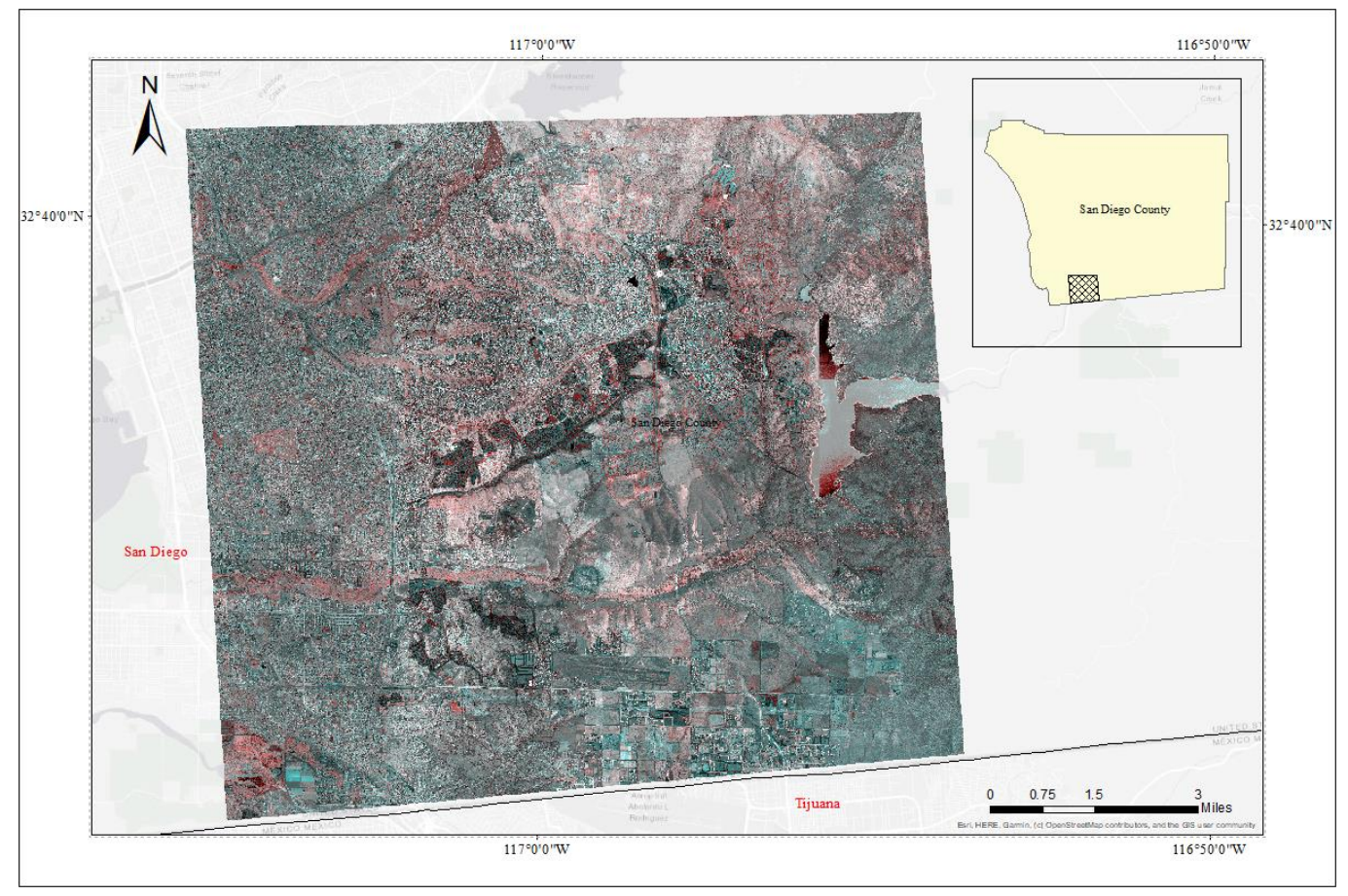

Figure 1: Study area within southern San Diego County, California, USA. Bi-temporal aerial orthoimage layerstack with Red image difference band displayed in the red colour plane and near-infrared (NIR) difference in the blue and green colour planes. Areas represented by red and cyan portray land-cover and land-use changes.

The specifications of bi-temporal aerial orthoimages obtained from the San Diego Association of Governments (SANDAG) and the National Agriculture Imagery Program (NAIP) are given in Table 1. The SANDAG imagery was captured between June and September 2000 with a positional accuracy similar to the USGS DOQQs (+/- $33 \mathrm{feet} ; 10 \mathrm{~m}$ ). The NAIP imagery was captured between April and August 2016 and met the accuracy requirement of $<4 \mathrm{~m}$ upon generation of mosaicked digital orthophoto quarter quadrangles. Spectral-radiometric data from the two ortho-image sets are uncalibrated 8-bit integer digital number (DN) values. Only the two-date Red and NIR bands were used to build multi-temporal image layerstacks for this study. Toure et al. (2013) demonstrated that using these two wavebands achieves high 
accuracy in single-date LU mapping based on GEOBIA with histogram-matching classifiers, without the high computational cost of using other, highly correlated, visible wavebands.

Table 1: Image characteristics

\begin{tabular}{llllll}
\hline $\begin{array}{l}\text { Image } \\
\text { Source }\end{array}$ & $\begin{array}{l}\text { Original } \\
\text { GSD }\end{array}$ & $\begin{array}{l}\text { Reprojected } \\
\text { GSD }\end{array}$ & $\begin{array}{l}\text { Spectral } \\
\text { Bands }\end{array}$ & $\begin{array}{l}\text { Radiometric } \\
\text { Quantization } \\
\text { (bit) }\end{array}$ & $\begin{array}{l}\text { Year of } \\
\text { Acquisition }\end{array}$ \\
\hline SANDAG & $0.6 \mathrm{~m}$ & $1 \mathrm{~m}$ & Red, NIR & 8 & 2000 \\
NAIP & $0.6 \mathrm{~m}$ & $1 \mathrm{~m}$ & Red, NIR & 8 & 2016 \\
\hline
\end{tabular}

Two-dates (2000 and 2016) of parcel-level land-use layers acquired from SANDAG (Table 2) provided the basis of the classification system (Table 3) and were used to generate LUC polygons for training and testing purposes. The classes of interest are general analytical LUC classes. These are divided into subclasses representing variations in land-cover composition within land-use polygons because of differences in age of development, building materials, etc. Subclass polygons served as thematic layers to assist the selection of parameter settings (i.e. Scale parameter for segmentation using eCognition GEOBIA software), and as a reference layer to refine the shape of resultant image objects manually.

Table 2: Land-use classification scheme

\begin{tabular}{ll}
\hline Specific Analytical LU Classes & SANDAG LU Code and Description \\
\hline Single-Family Residential (SFR) & 1100 Single-Family Residential \\
Multi-Family Residential (MFR) & 1200 Multi-Family Residential \\
Light Industry (LIND) & 2103 Light Industry - General \\
Commercial Development (COM) & 5002 Regional Shopping Centre \\
& 5003 Community Shopping Centre \\
& 5004 Neighbourhood Shopping Centre \\
Undeveloped (UNDEV) & 9101 Vacant and Undeveloped Land \\
\hline
\end{tabular}

Table 3: Land-use change and no-change classification scheme

\begin{tabular}{lll}
\hline & $\begin{array}{l}\text { General Analytical } \\
\text { LU Transition } \\
\text { Type of object }\end{array}$ & Subclasses \\
\hline Change & $\begin{array}{l}\text { UNSFR, Undeveloped } \\
\text { Land to Single- } \\
\text { Family Residential }\end{array}$ & $\begin{array}{l}\text { saUNSF, Undeveloped (cleared for } \\
\text { construction) to Single-Family } \\
\text { Residential }\end{array}$ \\
& - $\begin{array}{l}\text { sbUNSFR, Undeveloped (dry and grassy } \\
\text { land) to Single-Family Residential }\end{array}$
\end{tabular}


- ScUNSFR, Undeveloped (cleared for construction) to Single-Family Residential

- $\quad$ sdUNSFr, Undeveloped (soil, subshrub, small trees and trails) to SingleFamily Residential

\begin{tabular}{|c|c|}
\hline $\begin{array}{l}\text { UNMFR, Undeveloped } \\
\text { to Multi-Family } \\
\text { Residential }\end{array}$ & 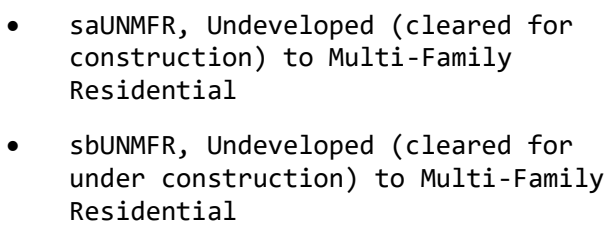 \\
\hline
\end{tabular}

\begin{tabular}{|c|c|}
\hline $\begin{array}{l}\text { UNLIND, Undeveloped } \\
\text { to Light Industry }\end{array}$ & $\begin{array}{l}\text { - } \quad \text { saUNLIND, Undeveloped (dry and grassy } \\
\text { land) to Light Industry } \\
\text { - } \quad \text { sbUNLINDL, Undeveloped (cleared for } \\
\text { under construction) to Light Industry }\end{array}$ \\
\hline $\begin{array}{l}\text { UNCOM, Undeveloped } \\
\text { Land to Commercial } \\
\text { Development }\end{array}$ & $\begin{array}{l}\text { - saUnCoM, Undeveloped (cleared for } \\
\text { construction) to Commercial } \\
\text { Development }\end{array}$ \\
\hline
\end{tabular}

No Change No Change

- NCSFR, No-change Single-Family Residential

- $\quad$ NCMFR, No-change Multi-Family Residential

- NCLIND, No-change Light Industry

- NCCOM, No-change Commercial Development

\section{Methodology}

A sequence of image-processing and analysis procedures were applied to delineate and identify transition classes for LUC objects using R, ArcGIS Pro, eCognition and Excel software packages, as shown in Figure 2. The general procedures consist of five steps: (1) image preprocessing, (2) segmentation, (3) generating training and testing histograms and regions of interest, (4) classification, and (5) classification accuracy statistics.

\subsection{Image pre-processing}

ArcGIS Pro software was used to perform image pre-processing to standardize characteristics of aerial orthoimages. Both images were subset to the extent of the study area, co-registered, and reprojected to $1 \mathrm{~m}$ GSD with UTM coordinates. Two layerstack images were created: (1) composites of bi-temporal Red and NIR bands; (2) temporal-spectral differences (time2 time1) of Red and NIR wavebands. 


\subsection{Segmentation and selection of regions of interest}

Potential temporal-spectral image objects (i.e. regions of interest, or ROIs) for training and testing were delineated by segmentation of two multi-temporal orthoimagery layerstacks using the multi-resolution segmentation algorithm in eCognition software. After trial and error, the segmentation Scale parameters ranged between 200 and 300 per class. We segmented finescale objects and then merged the resulting segments into larger image objects. The final segmentation layer was overlaid with polygons representing LUC classes derived from the reference GIS layer, to enable delineation of ROIs used to extract training data (e.g. pixels representing known LUC objects and used for training classifiers). Image objects were manually edited (i.e. polygons were split and merged) during the refinement process. ROIs of LUC subclasses were delineated based on the refined image objects and the reference LUC class layer. The number of training and testing ROIs per subclass was determined from the resulting ROIs by visual image interpretation (Table 4). These ROIs were utilized to extract histogram signatures (i.e. frequency distribution of within-object digital numbers) using the Zonal Histogram tool in ArcGIS Pro, and applied to nearest-neighbour classification using eCognition.

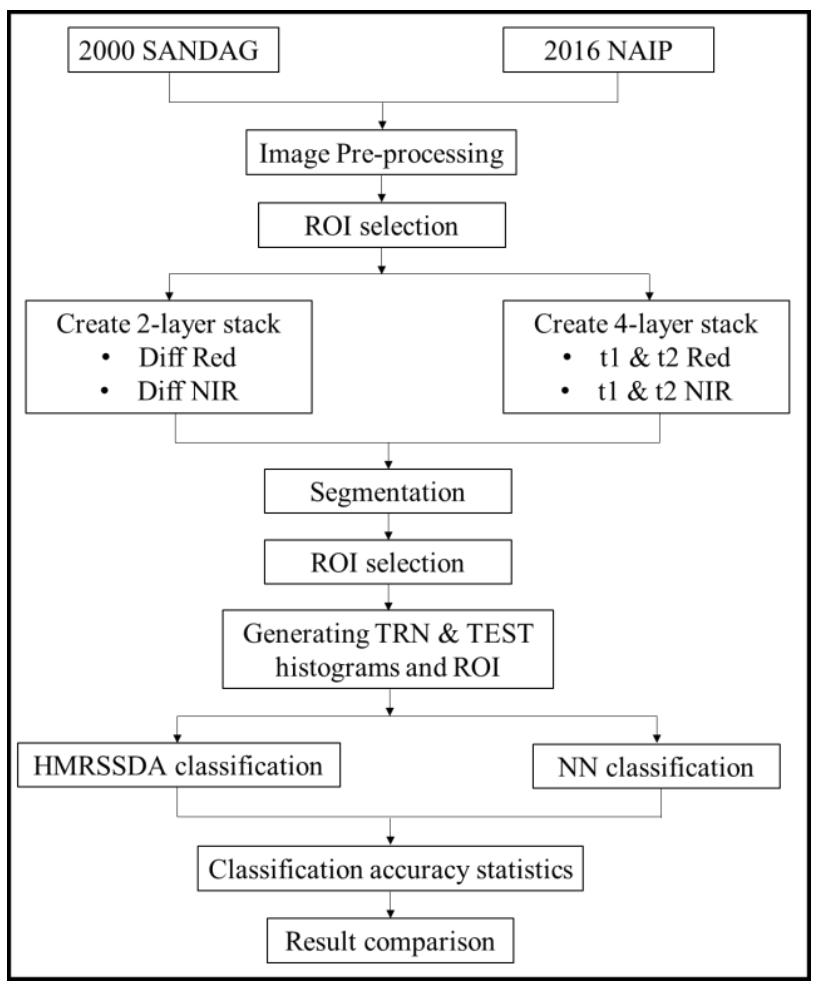

Figure 2: Processing flow. Diff Red and Diff NIR stand for temporal-spectral Red and NIR wavebands respectively. SANDAG = San Diego Association of Governments; NAIP = National Agriculture Imagery Program; $\mathrm{ROI}=$ regions of interest; $\mathrm{TRN}=$ testing; $\mathrm{TEST}=$ testing; $\mathrm{NN}=$ nearest neighbour; HMRSSDA = histogram matching root sum squared differential area. 


\subsection{Generating training and testing histograms and regions of interest}

Training and testing histograms for each subclass were evaluated by plotting histogram signature curves. The histogram curves that consistently represented the curve patterns for within-class LUC objects were used to compute mean training histograms for each subclass based on three different mathematical measures of means (arithmetic, geometric and Pythagorean). Three different mean values were computed for layerstacks with bi-temporal and bi-spectral feature inputs as follows:

$$
\begin{aligned}
& \text { Arithmetic Mean }=(\mathrm{t} 1 \mathrm{NIR}+\mathrm{t} 1 \mathrm{RED}+\mathrm{t} 2 \mathrm{NIR}+\mathrm{t} 2 \mathrm{RED}) / 4 \\
& \text { Geometric Mean }=\sqrt[4]{\mathrm{t} 1 \mathrm{NIR} \times \mathrm{t} 1 \mathrm{RED} \times \mathrm{t} 2 \mathrm{NIR} \times \mathrm{t} 2 \mathrm{RED}} \\
& \text { Pythagorean Mean }=\sqrt[4]{\mathrm{t}^{\mathrm{N} I R} \mathrm{R}^{4}+\mathrm{t} 2 \mathrm{RED}^{4}+\mathrm{t} 2 \mathrm{NIR}^{4}+\mathrm{t} 2 \mathrm{RED}^{4}}
\end{aligned}
$$

For layerstacks with temporal-spectral difference bands, mean values were calculated as follows:

$$
\begin{aligned}
\text { Arithmetic Mean } & =(\text { Diff NIR }+ \text { DIFF RED }) / 2 \\
\text { Geometric Mean } & =\sqrt{\text { Diff NIR } \times \text { Diff RED }} \\
\text { Pythagorean Mean } & =\sqrt{\text { Diff NIR }^{2}+\text { Diff RED }^{2}}
\end{aligned}
$$

where $\mathrm{t} 1=$ time $1, \mathrm{t} 2=$ time 2, Diff $=$ temporal-spectral difference band, NIR = nearinfrared red.

Histogram curves were averaged by the arithmetic method to generate a mean testing histogram for each within-class LUC object. Their corresponding image objects also served as training and testing ROIs for nearest-neighbour classification; resultant mean training and testing histograms were applied in histogram curve classification.

Table 4: Number and area coverage of training and testing ROls per subclass

\begin{tabular}{cccccc}
\hline $\begin{array}{c}\text { LU Transition } \\
\text { Subclasses }\end{array}$ & $\begin{array}{c}\text { No. TRN } \\
\text { ROI }\end{array}$ & $\begin{array}{c}\text { Area Covered by } \\
\text { TRN ROI }\left(\mathrm{km}^{2}\right)\end{array}$ & $\begin{array}{c}\text { No. TEST } \\
\text { ROI }\end{array}$ & $\begin{array}{c}\text { Area Covered by } \\
\text { TEST ROI }\left(\mathrm{km}^{2}\right)\end{array}$ & $\begin{array}{c}\text { No. TOTAL } \\
\text { ROI }\end{array}$ \\
\hline SaUNSFR & 8 & 0.04 & 48 & 0.23 & 56 \\
SbUNSFR & 7 & 0.03 & 41 & 0.15 & 48 \\
SCUNSFR & 10 & 0.04 & 52 & 0.19 & 62 \\
SdUNSFR & 9 & 0.04 & 31 & 0.12 & 40 \\
SaUNMFR & 3 & 0.01 & 14 & 0.05 & 17 \\
SbUNMFR & 4 & 0.01 & 20 & 0.05 & 24 \\
SaUNLIND & 3 & 0.03 & 10 & 0.10 & 13 \\
SbUNLIND & 3 & 0.01 & 10 & 0.03 & 13 \\
SaUNCOM & 4 & 0.05 & 9 & 0.11 & 13 \\
NCSFR & 34 & 0.27 & 115 & 0.96 & 149 \\
NCMFR & 8 & 0.05 & 49 & 0.51 & 57 \\
NCLIND & 6 & 0.06 & 16 & 0.28 & 22 \\
NCCOM & 9 & 0.05 & 26 & 0.40 & 35 \\
\hline
\end{tabular}




\subsection{Histogram curve classification}

Histogram Matching Root Sum Squared Differential Area (HMRSSDA) was adopted to measure histogram similarity of LUC and no-change objects. The HMRSSDA calculation is derived as (Stow et al. 2012):

$$
\text { HMRSSDA }=1-\sqrt{\left(\sum_{\mathrm{i}=\mathrm{DN}_{\min }}^{\mathrm{n}=\mathrm{DN}_{\max }}\left(\mathrm{FS}_{\mathrm{i}}-\mathrm{FR}_{\mathrm{i}}\right)^{2}\right)}
$$

where FS $i=$ frequency of the mean testing histogram at bin $i=D N, F R i=$ frequency of the mean training histogram at bin $i=D N$, and $D N=$ digital number.

The total number of pixels in each LUC/no-change object was divided by frequency count at each histogram bin for the normalization of bin values, to minimize the possible influence on varying object sizes (i.e. numbers of within-object pixels).

The accuracy of the HMRSSDA classifier was tested based on the layerstack change analysis approach within a GEOBIA framework using a set of R scripts and Excel. The hybrid of feature inputs (i.e. Diff_NIR and Diff_RED combined, or the t1NIR, t1RED, t2NIR and t2RED combined) provides information that can yield high classification accuracy (Toure et al. 2013).

\subsection{Nearest-neighbour classification}

The classification of layerstack images with the nearest-neighbour classifier was conducted using eCognition. Nearest-neighbour classification in eCognition computes the feature space distance from the testing object to each of the training objects as follow (eCognition Developer (2014):

$$
\text { Feature Space Distance }(\mathrm{d})=\sqrt{\sum_{\mathrm{f}}\left(\frac{\mathrm{v}_{\mathrm{f}}(\mathrm{s})-\mathrm{v}_{\mathrm{f}}(\mathrm{o})}{\sigma_{\mathrm{f}}}\right)}
$$

where $d=$ distance between the testing object $(s)$ to the training object $(o) ; v f(s)=$ feature value of training object for feature $(f) ; v f(0)=$ feature value of testing object for feature $(f)$; of = standard deviation of the feature values for feature $(f)$. The testing objects are assigned to the class of the training object having the shortest distance.

\subsection{Classification accuracy assessment}

Classification accuracy metrics based on subclasses were created using $\mathrm{R}$ programming scripts. The metrics were aggregated to a more general LUC class level which includes the targeted change and no-change classes for this study (Table 5). Overall classification accuracy for all objects, change and no-change objects, as well as producer's and user's accuracy are documented for each of the metrics. Since the goal of this study was to classify general analytical LUC classes, the resultant accuracy metrics were restructured by aggregation of subclasses to general LUC and no-change classes. 
Table 5: Number and area coverage of training and testing regions of interest per general class

\begin{tabular}{cccccc}
\hline $\begin{array}{c}\text { LU } \\
\text { Subsition }\end{array}$ & $\begin{array}{c}\text { No. TRN } \\
\text { ROI }\end{array}$ & $\begin{array}{c}\text { Area Covered } \\
\text { by TRN ROI } \\
(\mathrm{km2})\end{array}$ & $\begin{array}{c}\text { No. } \\
\text { TEST } \\
\text { ROI }\end{array}$ & $\begin{array}{c}\text { Area Covered by } \\
\text { TEST ROI (km2) }\end{array}$ & $\begin{array}{c}\text { No. TOTAL } \\
\text { ROI }\end{array}$ \\
\hline UNSFR & 34 & 0.15 & 172 & 0.70 & 206 \\
UNMFR & 7 & 0.02 & 34 & 0.10 & 41 \\
UNLIND & 6 & 0.05 & 20 & 0.13 & 26 \\
UNCOM & 4 & 0.05 & 9 & 0.11 & 13 \\
No Change & 57 & 0.43 & 206 & 2.14 & 263 \\
\hline $\begin{array}{l}\text { Total extent of the study } \\
\text { TEST = testing }\end{array}$ & & &
\end{tabular}

\section{Results}

\subsection{Classifier influence}

The HMRSSDA (histogram) classifier consistently yielded higher classification accuracy compared to the nearest-neighbour classifier for identification of change and no-change objects, no matter which feature inputs or approaches to combining multiple-feature classification measures were applied, as shown in Tables 6-8. The combination of HMRSSDA with arithmetic mean and temporal-spectral difference bands yielded the highest accuracies: $78.72 \%$ overall accuracy for change objects and $100 \%$ for no-change objects.

Table 6: Overall accuracy results for arithmetic mean

\begin{tabular}{|c|c|c|c|c|}
\hline \multirow{2}{*}{$\begin{array}{l}\text { Classifier } \\
\text { Layerstack Input }\end{array}$} & \multicolumn{2}{|c|}{ HMRSSDA } & \multicolumn{2}{|c|}{ Nearest Neighbour } \\
\hline & Diff NIR & t1NIR & Diff NIR & t1NIR \\
\hline & Diff RED & t1RED & Diff RED & t1RED \\
\hline & & t2NIR & & t2NIR \\
\hline & & t2RED & & t2RED \\
\hline Overall_Accuracy (ALL) & 79.82 & 76.19 & 60.09 & 74.15 \\
\hline Overall_Accuracy (TRANS) & 78.72 & 60.85 & 48.09 & 69.36 \\
\hline Overall_Accuracy (NC) & 81.07 & 93.69 & 73.79 & 79.61 \\
\hline
\end{tabular}

\subsection{Influence of type of mean calculation}

Using arithmetic and Pythagorean mean for combining classification measures of multiple histogram-matching features (e.g. spectral bands), $77 \%$ of change objects were successfully identified, which is 8 to $9 \%$ higher than when using the geometric mean. For no-change objects, arithmetic and geometric means achieved the highest (93\% and 100\%) classification 
accuracy, which is about 6\% higher than with the Pythagorean mean (87.38\%). Additionally, classifications based on the arithmetic mean show the smallest difference between the highest and the lowest accuracies for change and no-change objects individually. The accuracy of only one classification product (48.09\%) is below $60 \%$ and most are well over $70 \%$, suggesting that the arithmetic mean is preferable to the other two mean inputs.

\subsection{Feature input influence}

For most of the feature combinations, the layerstack feature inputs containing temporalspectral difference bands generally outperformed the layerstack approach with bi-temporal spectral bands in classifying change objects. However, the opposite resulted when classifying no-change objects. The temporal-spectral difference bands resulted in the highest classification accuracy for change $(78.72 \%)$ and no-change $(87.38 \%)$ objects, compared to $69.36 \%$ and $100 \%$ accuracy yielded with bi-temporal spectral bands. This suggests that histograms of temporalspectral difference bands contained more distinctive change signals than the histograms associated with bi-temporal spectral bands. The layerstack that has bi-temporal spectral bands uses full histogram information from each band input, which intensifies no-change histogram signatures; therefore, no-change objects were generally well-differentiated.

Table 7: Overall accuracy results for geometric mean

\begin{tabular}{lcccc}
\hline Classifier & \multicolumn{2}{c}{ HMRSSDA } & \multicolumn{2}{c}{ Nearest Neighbour } \\
\cline { 2 - 5 } Layerstack Input & Diff NIR & t1NIR & Diff NIR & t1NIR \\
& Diff RED & t1RED & Diff RED & t1RED \\
& & t2NIR & & t2NIR \\
& & t2RED & & t2RED \\
Overall_Accuracy (ALL) & 70.29 & 71.20 & 53.06 & 45.35 \\
Overall_Accuracy (TRANS) & 69.79 & 45.96 & 43.40 & 36.17 \\
Overall_Accuracy (NC) & 70.87 & 100.00 & 64.08 & 55.83 \\
\hline ALL = all testing objects; TRANS = change objects; NC $=$ no-change objects
\end{tabular}

Table 8: Overall accuracy results for Pythagorean mean

\begin{tabular}{lcccc}
\hline Classifier & \multicolumn{2}{c}{ HMRSSDA } & \multicolumn{2}{c}{ Nearest Neighbour } \\
\cline { 2 - 5 } Layerstack Input & Diff NIR & t1NIR & Diff NIR & t1NIR \\
& Diff RED & t1RED & Diff RED & t1RED \\
& & t2NIR & & t2NIR \\
& & t2RED & & t2RED \\
Overall_Accuracy (ALL) & 69.84 & 43.99 & 53.74 & 46.49 \\
Overall_Accuracy (TRANS) & 54.47 & 12.34 & 43.83 & 39.15 \\
Overall_Accuracy (NC) & 87.38 & 80.10 & 65.05 & 54.85 \\
\hline ALL = all testing objects; TRANS $=$ change objects; NC $=$ no-change objects
\end{tabular}




\subsection{Most accurate combination of inputs and classifier}

Overall, the change-identification approach yielding the highest accuracy for histogram classification is the combination of HMRSSDA, arithmetic mean and the layerstack that has temporal-spectral difference bands. For nearest-neighbour classification, the most accurate approach is the combination with arithmetic mean and the layerstack that has bi-temporal spectral bands. Classification performance for each general LUC class is shown in Tables 9 and 10. Among the change classes, the HMRSSDA approach classified UNSFR most accurately $(82.56 \%)$, and UNLIND least accurately $(65 \%)$. The nearest-neighbour method classified UNSFR most accurately (75.58\%), and UNCOM least accurately (44.44\%). For the no-change class, both approaches produced similar accuracy, of around $80 \%$, but the HMRSSDA is $1.61 \%$ higher than for the nearest-neighbour classifier.

The HMRSSDA classification yielded $79.82 \%$ overall accuracy, with 39 change objects misclassified to no-change, and only seven no-change objects misclassified to change classes (i.e. UNSFR, UNMFR, UNLIND and UNCOM). The nearest-neighbour classification resulted in $74.15 \%$ overall accuracy, with 42 change objects incorrectly classified to no-change, and 52 no-change objects allocated to the LUC classes. This suggests that the nearestneighbour classification over-classified change and no-change objects, while the HMRSSDA classification limited such over-classification of change objects (52 to 7) substantially, and slightly for no-change objects (42 to 39).

Table 9: Histogram classification accuracy matrix for the combination of HMRSSDA, arithmetic mean, and layerstack having temporal-spectral difference bands.

\begin{tabular}{|c|c|c|c|c|c|c|c|c|}
\hline & & \multicolumn{6}{|c|}{ Reference Data } & \multirow[b]{2}{*}{ User's } \\
\hline & & UNSFR & UNMFR & UNLIND & UNCOM & No Change & Total & \\
\hline \multirow{7}{*}{ 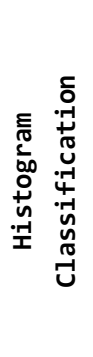 } & UNSFR & 142 & 9 & 7 & 0 & 27 & 185 & 76.76 \\
\hline & UNMFR & 20 & 24 & 0 & 1 & 0 & 45 & 53.33 \\
\hline & UNLIND & 5 & 0 & 13 & 1 & 10 & 29 & 44.83 \\
\hline & UNCOM & $\theta$ & 0 & 0 & 6 & 2 & 8 & 75.00 \\
\hline & No Change & 5 & 1 & 0 & 1 & 167 & 174 & 95.98 \\
\hline & Total & 172 & 34 & 20 & 9 & 206 & 441 & \\
\hline & Producer's & 82.56 & 70.59 & 65.00 & 66.67 & 81.07 & & \\
\hline
\end{tabular}

Overall accuracy, 352/441=79.82\%; User's = user's accuracy; Producer's = producer's accuracy 
Table 10: Nearest-neighbour classification accuracy matrix for the combination of NN, arithmetic mean, and layerstack having bi-temporal spectral bands.

\begin{tabular}{|c|c|c|c|c|c|c|c|c|}
\hline & & \multicolumn{6}{|c|}{ Reference Data } & \multirow[b]{2}{*}{ User's } \\
\hline & & UNSFR & UNMFR & UNLIND & UNCOM & $\begin{array}{l}\text { No } \\
\text { Change }\end{array}$ & Total & \\
\hline \multirow{7}{*}{ 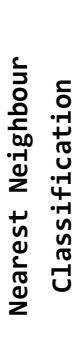 } & UNSFR & 130 & 6 & 2 & 0 & 29 & 167 & 77.84 \\
\hline & UNMFR & 5 & 19 & 1 & 1 & 6 & 32 & 59.38 \\
\hline & UNLIND & 3 & 1 & 10 & 1 & 2 & 17 & 58.82 \\
\hline & UNCOM & 0 & 0 & 0 & 4 & 5 & 9 & 44.44 \\
\hline & No Change & 34 & 8 & 7 & 3 & 164 & 216 & 75.93 \\
\hline & Total & 172 & 34 & 20 & 9 & 206 & 441 & \\
\hline & Producer's & 75.58 & 55.88 & 50.00 & 44.44 & 79.61 & & \\
\hline
\end{tabular}

Overall accuracy, 327/441=74.15\%; User's = user's accuracy; Producer's = producer's accuracy

\section{Discussion and Conclusion}

The objective of this study was to develop, test and compare multi-temporal image change identification approaches through GEOBICA techniques that use histogram and nearestneighbour classification with various feature inputs, in the context of updating extant land-use GIS layers that become out of date as a result of urban growth. In such a context, an outdated land-use GIS layer could be updated based on multi-temporal images. The full information content of frequency distributions of pixels within LUC/no-change objects was evaluated by the feature inputs (i.e. layerstack, classifier and mean calculation). The resultant classification products depict five general classes (i.e. four LUC classes and one no-change class), which were created by aggregation of temporal-spectral subclasses.

The core (second) research question addressed in this study pertains to the comparative performance of a histogram and a nearest-neighbour classifier. Classifier influence was evaluated, and we found that the histogram classifier consistently outperformed the nearestneighbour classifier, with just two exceptions. The histogram classifier exploits the complex frequency distributions of the LUC objects better than the nearest-neighbour classifier, which answers the first research question. Figures 3 presents training (mean) histogram curves for (a) change and (b) no-change subclasses derived from the Red temporal-spectral difference band. The majority of LUC curves tend to be non-normally distributed, while the no-change objects have normally distributed curves. Overall, the shapes of the histogram curves for each subclass appear to be consistent for both Red and NIR temporal-spectral difference bands. 

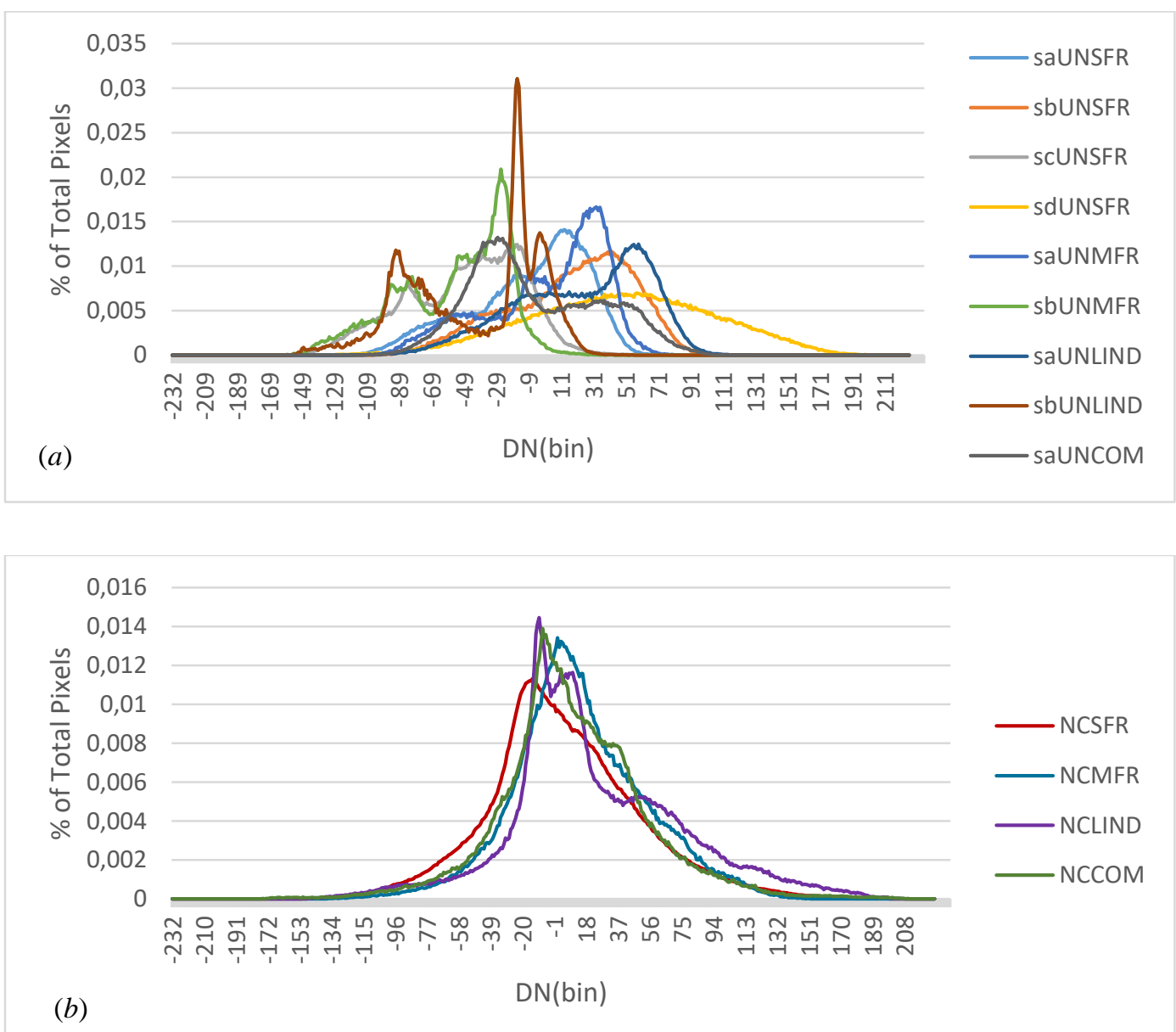

Figure 3: Training (arithmetic mean) histogram curves for (a) change and (b) no-change subclasses based on each RED temporal-spectral difference feature input.

Another research question pertains to which combination of feature inputs results in the most accurate identification of change and no-change objects, as well as what the classification accuracy of different types of LUC/no-change objects is. We demonstrate that the combination temporal-spectral difference bands, HMRSSDA and arithmetic mean is the most accurate change-identification approach. This combination identified UNSFR most accurately $(82.56 \%)$. UNLIND was classified least accurately $(65 \%)$. Compared to the most accurate nearest-neighbour classification combination (arithmetic mean and bi-temporal spectral bands), the HMRSSDA combination limited over-classification of change objects substantially, and slightly for no-change objects. The overall accuracy for all objects is $5.67 \%$ higher than the nearest-neighbour method.

A further research question was which type of mean calculation for combining histogram classification metrics and feature inputs yielded the highest change-identification accuracy. We found that the arithmetic mean $(78.72 \%)$ slightly outperformed the Pythagorean mean 
(77.87\%), followed by geometric mean $(69.79 \%)$. For no-change objects, the geometric mean resulted in the highest identification accuracy $(100 \%)$, followed by arithmetic mean $(93.69 \%)$ and Pythagorean mean (87.38\%).

For the question concerning feature input influences, the layerstack with temporal-spectral difference bands generally yielded more accurate identification of change objects. The highest identification accuracy was $78.72 \%$, which is $9.36 \%$ higher than the greatest accuracy using bitemporal spectral bands. Using a temporal-spectral layerstack yielded 100\% identification accuracy for no-change objects, which is $12.62 \%$ higher than the highest accuracy obtained using temporal-spectral difference bands.

While the histogram classifier was shown to more accurately identify change and no-change objects for our study area and context, some challenges and limitations need to be addressed. Histogram curve-matching classification may be challenging if the classification level is too detailed (e.g. land-use data at parcel level) or if the size of image objects is very small (e.g. land parcels). Greater effort would be required to train on LUC types that exhibit high within-class temporal-spectral variability, which would necessitate adding more subclasses for classification, and then aggregating subclasses post-classification. Another challenge is to find appropriate segmentation parameters (e.g. Scale and the composition of the homogeneity criterion) depending on class of interest, feature inputs, image resolution characteristics, and/or study regions.

Follow-on research should include testing the histogram classifier in other study sites/contexts with different LUC classes and using different image types. Developing significance tests for the histogram classifier is another topic for further investigation. Influences of spatial resolution, band inputs and quantization level (i.e. number of bins) should also be assessed. Finally, we recommend that the histogram classifier be tested for a post-classification comparison approach to GEOBIA-based LUC analysis.

\section{Acknowledgments}

Data Solutions of the San Diego Association of Governments (SANDAG) provided land-use data files used as reference data. Professors Sherry Ryan and Ming Tsou provided valuable feedback regarding the research design and results summary.

\section{References}

Anderson, J. R., Hardy, E. E., Roach, J. T., \& Witmer, R. E. (1976). A land use and land cover classification system for use with remote sensor data. In Professional Paper.

Blaschke, T., Hay, G. J., Kelly, M., Lang, S., Hofmann, P., Addink, E., Feitosa, R. Q.

(2014). Geographic Object-Based Image Analysis - Towards a new paradigm. ISPRS Journal of Photogrammetry and Remote Sensing 87, 180-91.

Chen, G., Hay, G. J., Carvalho, L. M. T., \& Wulder, M. A. (2012). Object-based change detection. International Journal of Remote Sensing 33 (14), 4434-57. 
Cleve, C., Kelly, M., Kearns, F. R., \& Moritz, M. (2008). Classification of the wildland-urban interface: A comparison of pixel- and object-based classifications using high-resolution aerial photography. Computers, Environment and Urban Systems 32 (4), 317-26.

Desclée, B., Bogaert, P., \& Defourny, P. (2006). Forest change detection by statistical object-based method. Remote Sensing of Environment 102 (1), 1-11.

eCognition Developer, T. (2014). 9.0 User Guide. Trimble Germany GmbH, Munich, Germany.

Gao, Y., \& Mas, J. F. (2008. A comparison of the performance of pixel-based and object-based classifications over images with various spatial resolutions. Online journal of earth sciences 2 (1), 27-35.

Hussain, M., Chen, D., Cheng, A., Wei, H., \& Stanley, D. (2013). Change detection from remotely sensed images: From pixel-based to object-based approaches. ISPRS Journal of Photogrammetry and Rremote Sensing 80, 91-106.

Im, J., Jensen, J. R., \& Tullis, J. A. (2008). Object-based change detection using correlation image analysis and image segmentation. International Journal of Remote Sensing 29 (2), 399-423.

Johansen, K., Arroyo, L. A., Phinn, S., \& Witte, C. (2010). Comparison of geo-object based and pixelbased change detection of riparian environments using high spatial resolution multi-spectral imagery. Photogrammetric Engineering \& Remote Sensing 76 (2), 123-36.

King, D. J. (2011). Comparison of pixel- and object-based classification in land cover change mapping AU - Dingle Robertson, Laura. International Journal of Remote Sensing 32 (6), 1505-29.

Stow, D. (2010). Geographic object-based image change analysis. In Handbook of applied spatial analysis (pp. 565-582). Springer, Berlin, Heidelberg.

Stow, D. A., Toure, S. I., Lippitt, C. D., Lippitt, C. L., \& Lee, C.-R. (2012). Frequency distribution signatures and classification of within-object pixels. International Journal of Applied Earth Observation and Geoinformation 15, 49-56.

Toure, S. I., Stow, D. A., Weeks, J. R., \& Kumar, S. (2013). Histogram curve matching approaches for object-based image classification of land cover and land use. Photogrammetric Engineering \& Remote Sensing 79 (5), 433-40.

Whiteside, T. G., Boggs, G. S., \& Maier, S. W. (2011). Comparing object-based and pixel-based classifications for mapping savannas. International Journal of Applied Earth Observation and Geoinformation 13 (6), 884-93.

Zhou, W., Troy, A., \& Grove, M. (2008). Object-based land cover classification and change analysis in the Baltimore metropolitan area using multitemporal high resolution remote sensing data. Sensors 8 (3), 1613-36. 